\title{
Educating Health Professionals about the Electronic Health Record (EHR): Removing the Barriers to Adoption
}

\section{Elizabeth Borycki*}

School of Health Information Science

University of Victoria

Victoria, British Columbia, Canada

E-mail: emb@uvic.ca

\section{Ronald S. Joe}

School of Health Information Science

University of Victoria

Victoria, British Columbia, Canada

E-mail: ronjoe@shaw.ca

\section{Brian Armstrong}

School of Health Information Science University of Victoria

Victoria, British Columbia, Canada

E-mail: brian.armstrong@ehrchitect.com

\section{Paule Bellwood}

School of Health Information Science University of Victoria Victoria, British Columbia, Canada

E-mail: paulebw@uvic.ca

\section{Rebecca Campbell}

School of Health Information Science

University of Victoria

Victoria, British Columbia, Canada

E-mail: recamp@uvic.ca

*Corresponding author

\begin{abstract}
In the healthcare industry we have had a significant rise in the use of electronic health records (EHRs) in health care settings (e.g. hospital, clinic, physician office and home). There are three main barriers that have arisen to the adoption of these technologies: (1) a shortage of health professional faculty who are familiar with EHRs and related technologies, (2) a shortage of health informatics specialists who can implement these technologies, and (3) poor access to differing types of EHR software. In this paper we outline a novel
\end{abstract}


solution to these barriers: the development of a web portal that provides facility and health professional students with access to multiple differing types of EHRs over the WWW. The authors describe how the EHR is currently being used in educational curricula and how it has overcome many of these barriers. The authors also briefly describe the strengths and limitations of the approach.

Keywords: Electronic health record; Electronic patient record; Personal health record; Medical education; Nursing education; Health informatics education; Biomedical informatics education; Adoption

Biographical notes: Elizabeth Borycki is an Assistant Professor at the School of Health Information Science at the University of Victoria. She has extensive expertise in the area of health informatics and holds a $\mathrm{PhD}$ from the University of Toronto.

Ronald Joe is a practicing physician who has developed a range of electronic health record applications for use in practice and for education. He holds a Masters in Health Information Science from the University of Victoria.

Brian Armstrong is a consultant with extensive experience in the development and hosting of electronic health record systems.

Paule Bellwood is a research assistant in the School of Health Information Science at the University of Victoria, Canada.

Rebecca Campbell is a research assistant in the School of Health Information Science at the University of Victoria, Canada.

\section{Introduction}

Electronic health records are being implemented world-wide by governments (i.e. in the Americas, Europe, Asia, and Africa) in an effort to improve the quality, efficiency and effectiveness of healthcare delivery globally (Borycki \& Kushniruk, 2008; Shortliffe \& Cimino, 2008; World Health Organization, 2005). Within countries and healthcare systems the introduction and implementation of electronic health records (EHRs) or lifetime respositories of an individual's health information is a widespread phenomena that will have an impact upon all aspects of healthcare settings (i.e. hospitals, nursing homes, physician offices, clinics and home care) (Kushniruk \& Borycki, 2008; Shorliffe and Cimino, 2008; World Health Organization, 2005). As a result, there has emerged a new need to educate health professionals (e.g. physicians, nurses) about these health information systems and their associated devices that health professionals (e.g. physicians, nurses, social workers) will use when providing patient care. Unfortunately, health professional educational programs (e.g. medical, nursing, social work) have not kept up with these advancements in real-world healthcare settings. There are a few health professional educational programs that offer student's opportunities to learn about these differing types of health information systems in classroom settings prior to working in the real-world (Borycki et al., 2009).

There are a number of underlying reasons for health professional academic programs being unable to provide students with opportunities to learn about how to use these technologies before encountering them in real-world healthcare settings (Borycki et al., 2009), including shortages of health professional faculty with competencies in this 
area and health informatics professionals who can implement these systems. Furthermore, there are few cost-effective technological solutions that can provide geographically distant students (i.e. those located in different parts of a province, state or region) with the breadth and depth of technology-based experiences needed to use EHRs in clinical settings. There is a need to provide a background in the theoretical underpinnings of the design, development and use of these technologies to give students an understanding and lifelong ability to work in technology-enabled healthcare settings. (Armstrong et al., 2009; Borycki et al., 2009). Therefore, in this paper we: (1) provide an overview of the technological context and a tool for integrating these technologies into health professional curricula, (2) describe some of the barriers currently encountered by health professional academic programs in providing students with the opportnity to learn about these technologies, (3) describe a real-world solution, and (4) describe our initial experiences in using this technology to overcome these barriers to integrating EHRs into health professional student education. We begin this paper by providing some background information about EHRs.

\section{Background}

\subsection{Emergence of Electronic Health Records as a Dominant Technology in Healthcare}

Over the past two decades EHRs have emerged as the dominate information technology in healthcare. EHRs are being used by governments and regional healthcare administrators to modernize and improve the quality, efficiency, effectiveness and safety of healthcare delivery systems (Nohr \& Boye, 2008). EHRs are being rapidly implemented across the globe in many countries (e.g. United Kingdom, France, Germany, Canada, United States, Australia etc.) (Protti, 2007). EHRs are electronic repositories of patient data that allow physicians to electronically enter and retrieve data provide, decision support, and support the linking of patient data across regions. Worldwide there has been an exponential rise in the number of EHR implementations and the number of health professionals that are using EHRs and their components in patient care (Borycki et al., 2009). Research has suggested that in the next 20-30 years EHRs and their components will be a source of patient and citizen health information in many healthcare settings. According to many global agencies (including the World Health Organization) EHRs will be the primary technology platform to be used in managing and documenting citizen and patient health and healthcare worldwide (Nohr \& Boye, 2008; Protti, 2007; World Health Organization, 2005).

In response to this global rise in the number of EHRs that are being used, there has emerged a significant need to provide health professionals (e.g. physicians, nurses etc.) with the appropriate education so that they may learn about this technology, before they are required to use it in real-world settings. This is especially important given the current research that suggests that poor health professional understanding of EHRs may lead to poor interactions between the healthcare profesional, the patient and the technology and in turn may lead to medical errors (Borycki \& Kushniruk, 2008; Koppel et al., 2005; Kushniruk et al., 2005; Patel et al., 2001). There are many EHRs (and their components - e.g. computerized physician order entry) being developed and/or sold in the healthcare marketplace. Many of these EHRs and their components have differing 
interface designs, features and functions that make them unique, but they also have many similarites among them (Borycki et al., 2009).

In general EHRs can be classified according to the purpose and setting of use (Nagle, 2007; Shortliffe \& Cimino, 2008). At present there are four main types of records that are considered foundational to modernizing the healthcare system using technology. These EHRs include: (1) electronic patient records or EPRs, (2) electronic medical records or EMRs, (3) personal health records or PHRs, and (4) electronic health records (EHRs) when they are used as respositories for a citizen's lifetime information (Kushniruk \& Borycki, 2008; Shortliffe \& Cimino, 2008). To better understand how these records work in a healthcare system, the authors provide an overview of their primary functions and setting of use.

Electronic patient records and electronic medical records are the most ubiquitous types of EHRs in healthcare systems. Electronic patient records are those records used by members of the health care team in hospitals and nursing homes who are involved in the patient's care (Nagle, 2007). Alternatively, electronic medical records are used by physicians in their offices. They may also be used by physicians and other health professionals such as nurse practitioners, nurses, social workers and dieticians who work in clinics (Nagle, 2007). Personal health records refer to those records stored and used by patient's and their caregivers in their homes or on devices of their choosing (e.g. iPhones). Personal health records store information about a patient's health status, treatments and diagnoses (as recorded by a patient or a member of their family). These records may also include information the patient has obtained from electronic patient records or electronic medical records (Popkin et al., 2009). Lastly, EHRs are lifetime repositories of an individual's health information held at a regional, or country level (Nagel, 2004; Shorliffe \& Ciminio, 2008). Electronic patient records generated in a hospital and electronic medical records generated in a physician's office may or may not be linked to the EHR. In general governments plan to link electronic medical and electronic patient records to a citizen's EHR at some point in the future to create a lifetime repository of health information for each citizen. In summary each of these types of records are used in differing healthcare settings. Fundamentally, these records are very similar, but there are differences among them in terms of the types of health profesionals who use them (e.g. physicians only, or the full healthcare team), where they are used (e.g. hospital versus community), the type of information that is stored in them, who places it there, and their interface design features and functions. For the purpose of this paper, the authors will refer to each of these types of technologies as EHRs.

\subsection{The Current State of EHR Use In Health Professional Student Educational Programs}

A recent review of the health professional literature on EHR use in health professional education (including medicine, nursing, allied health and health informatics) has revealed that health professionals have very little exposure to differing types of EHRs (i.e. electronic patient records, electronic medical records, personal health records and EHRs) within the context of the classroom setting. Furthermore, there is little integration of EHRs throughout a traditional four year health professional educational curriculum in many countries (Borycki et al., 2009; Otto \& Kushniruk, 2009 ). In a few cases where health professionals learn about EHRs, they learn about this technology in one of three ways: (1) through lecture or laboratory work in a university or college, (2) in a university or college course on health informatics where they work with a single EHR as part of a lecture or laboratory course - where the student has exposure to only one type of a EHR - 
such as an EHR supplied by a single vendor, (3) during a real-world clinical practica or internship experience, where the health professional student is not only learning about how to manage a patient's care, but they are also learning about how to use the EHR to document and support their decision making (Borycki et al., 2009). For these students, this involves using one of these EHRs during a clinical practica under the supervision of a clinician (who directly supervises the student) after receiving training from a healthcare organization (e.g. a hospital) or from a senior health professional (e.g. physician, nurse or health informatics professional) who may be supervising their work.

This type of learning often does not provide the full breadth of information nor does it provide a student with an opportunity to develop a conceptual understanding of the EHR that is necessary to understand the use, strengths and weaknesses of the differing types of EHRs. Ideally, formal education should take place in the form of lecture, discussion, and hands on work with the differing types of EHRs in response to differing situations that are similar to those encountered in the real world. This formal education allows for the development of a conceptual and practical understanding of the theoretical underpinnings of EHR design, development, use, strengths and limitations of the technology. Such knowledge is key to understanding the linkages between the technology's design, features and functions and the activities it performs for a health professional (e.g. acting as conduit for team communication, supporting individual and health professional team decision making, and using embedded decision support tools such as alerts and reminders) (Kuziemsky et al., 2009). Furthermore, such knowledge would allow health professionals to understand the strengths and limitations of the technology. For example, health professionals who understand how EHR features and functions may facilitate medical errors (i.e. a technology-induced error) will be able to identify potential risks associated with a technology's use and report on these risks to the information technology management department of a hospital, a vendor, or a government body so that the technology can be modified to improve its safety (Borycki \& Kushniruk, 2008; Kushniruk et al., 2005).

Currently, health professional academic programs do not provide students with a solid grounding in these aspects of EHRs. It must be noted that even technology savvy students experience difficulty in using EHRs. They are often unaware of the supporting features and functions of these records. They are also unaware of their potential pitfalls (e.g. the ability to facilitate some types of errors). This influences student use of such systems in the clinical practice context and in some cases may lead to low adoption rates or ineffective use of the technology. Such knowledge is critical for students understanding of EHRs that are currently being used, are in the process of being implemented and are being developed for future use. More importantly, such knowledge is essential for students who will graduate to become health professional practitioners and will be exposed to differing types of EHRs over the course of a lifetime of practice as they move from one jurisdiction to another and the technology itself changes over time (i.e. much as pilots learn the theoretical underpinnings of flight and aircraft maintenance and design before they learn about the specifics of flying a particular type of aircraft). Such a knowledgeable group of future health professional practitioners will help technology designers and implementers to create more safe and supportive EHRs for health professional use (Borycki \& Kushniruk, 2008; Borycki et al., 2009). 


\section{Barriers to Integrating EHRs into Health Professional Education}

There are a number of barriers that prevent the integration of EHRs into health professional education. Three main barriers are: (1) a shortage of faculty who are familiar with differing types of EHRs, (2) a shortage of health informatics professionals who can implement such systems, and (3) a shortage of opportunities to access multiple differing types of EHRs within the context of a health professional student academic program.

\subsection{Shortage of Faculty Who are Familiar with EHRs}

Over the past several years there has been investment by governments in EHRs and this investment has focused on the development and implementation of these technologies in healthcare settings. Less emphasis has been placed upon extending the health informatics competencies of health professional faculty and providing faculty with opportunities to integrate these competencies into health professional curricula (Borycki et al., 2009). It is interesting and worthy to note that health professional program faculty are technology literate. Faculty frequently use educational technology to provide distance education (e.g. Moodle, Ellumninate $\left.{ }^{\circledR}\right)$ (Clarke \& Nagle, 2004). As well, technologies such as computer controlled patient simulators are used by faculty to support health professional student (e.g. medical and nursing) development of clinical knowledge, skills and judgment to be used with patients (Issenberg et al., 1999). Presently, health professional faculty have few opportunities to access EHR systems. Furthermore, in cases where universities or colleges have invested in EHRs, typically only one type of EHR is available to teach students. There is a need for faculty to develop EHR competencies so that they may be able to integrate these competencies into health professional curricula. There is also a need to provide faculty with access to EHRs so that educational programs can provide faculty with opportunities to learn about how to use these systems and integrate them into health professional student curricula. Only a few regions of countries have identified the need to invest in faculty so they can teach these competencies. Even fewer have invested in providing faculty and students with access to such tools (e.g. British Columbia in Canada). Such investment is necessary to ensure that health professional faculty can teach students how to develop EHR competencies (Borycki et al., 2009).

\subsection{Shortage of Health Informatics Specialists to Implement EHRs}

There exists a significant shortage of qualified health informatics professionals with sufficient health, information and management science knowledge that can implement EHRs to be used in health professional academic programs (Borycki et al., 2009; Canada's Health Informatics Association, 2010). Globally, there are too few undergraduate and graduate prepared health informatics professionals. There is a scarcity of these individuals who have knowledge of EHRs and their use in healthcare settings world wide. Public (e.g. regional health authority) and private sector (e.g. healthcare insurance companies) demands for these individuals are high. Colleges and universities are competing for this scarce human resource with private and public sector organizations. The ability of colleges and universities to implement and maintain EHRs for the purpose of educating student health professionals is limited by the scarcity of those who are most knowledgeable about developing, designing and implementing these technologies. There is a need to provide access to EHRs in such a way that a province or country draws few health informatics human resources away from public and private sector organizations who are implementing EHRs in physician offices, clinics, hospitals, nursing homes and public health agencies in governments and regional health authorities worldwide. 


\subsection{Poor Access to Differing Types of EHR Software}

Many software vendors are developing differing types of EHRs and their components (i.e. EHRs, EMRs, EPRs, PHRs). Many of these products are proprietary in nature. Vendors of these information systems can limit those who have access to these systems. It is argued by some that this is to prevent other vendors from learning about their EHRs and gaining a competitive advantage (Dess, Lumpkin, Eisner \& Perdis, 2009). Health professional faculty and students need opportunities to learn about the differing types of EHR designs for their work in health care settings. Students should be exposed to these differing systems as part of their educational programs to develop an understanding of how differing systems work and can be used in the process of patient care (i.e. where no real patient data or patients are affected by changes made to the record in the process of learning - in a safe environment) (Borycki et al., 2009). As well, there is a need for students to work with differing types of EHRs to learn about how these systems can be deployed or implemented in differing health care organizations (e.g. differing hospitals use differing terminologies and may have processes that need to be reflected in the EHR to ensure good health professional task-technology fit and cognitive-socio-technical fit) (Borycki \& Kushniruk, 2010). Current opportunities to work with differing EHRs are limited for health professional students - to the best of our knowledge, only a few undergradauate and graduate programs have attempted to incorporate EHRs and their components into their curricula (Borycki et al., 2009). Many health professional programs provide access to only one EHR (thereby exposing students to only one type of record and one type of EHR design with its associated features and functions) (e.g. Connors et al., 2002). Providing such limited access to EHRs is unlike that provided to other disciplines (e.g. business, computer science and engineering) who are afforded the opportunity to work with a full range of software and software designs before working with real or "live" data. These opportunities are afforded to students in other disciplines in order to practice their skills and prevent any unnecessary errors in the real world once they graduate (Borycki et al., 2009). Research has shown that the practice of skills in laboratory settings under simulated conditions leads to fewer errors when working in the real world. Issenberg (1999) has shown that the use of computerized mannequins to train medical students reduces the likelihood they will commit an error in a real-world setting when working with patients. Therefore health professional students need to work with many types of records before they graduate from their program of studies to prevent errors involving EHR use (Borycki et. al., 2009; Borycki \& Kushniruk, 2008).

\subsection{Shortage of Access to EHRs Over the World Wide Web}

Historically, health professional student education has involved classroom and hospital based work. With the introduction of technology and health professional academic programs attempting to leverage the knowledge of faculty at differing sites and across geographic distances, there has emerged a need to provide access to EHRs that can be used to educate health professional students across multiple sites and cities that are geographically distant (Borycki et al., 2009). For example, at the University of Victoria in Canada, graduate programs deliver many courses online using synchronous and asynchronous technologies (e.g. Moodle, Elluminate ${ }^{\circledR}$ ). As well, some universities are training health professional students using a multi-site format with video conferencing. For example, the University of British Columbia Medical School educates medical students across the province of British Columbia with sites in Victoria, Vancouver, Prince George and soon to open Kelowna. Lastly, students may be located throughout a country during parts of their education. For example, undergraduate and graduate 
students participate in cooperative education in some programs as a way of obtaining practice experience in real-world settings. Students may take part in cooperative education across Canada and internationally as is the case at the University of Victoria School of Health Information Science. Lastly, there is an increased need to provide software to students outside the classroom environment (i.e. home, library or laboratory environment) prior to classroom work or after leaving the classroom environment to complete assignments. For example, in our experience in working with health informatics students, access to EHRs was required prior to classroom lectures and after lectures as part of laboratory work (Borycki, et. al., 2009).

\subsection{Nature of Real-World Work: EHR Changes Over Time}

Today's health professional work environment (e.g. hospitals, clinics and physician offices) is in a constant stage of change. This is especially the case when one considers the impact of EHRs upon health professional work. For example, in North America, hybrid paper-electronic environments, where part of the patient record is paper based and part of it is electronic predominate in health care settings (Borycki et. al., 2009). This is a result of EHRs being in various stages of implementation. Health professional students need to learn how to work with EHRs now before they are fully implemented. Health professional students also need to learn how to work with technology that will change over time in addition to changes in content and functionality (e.g. much as the EHR changes in response to medical, nursing and other allied health practice). Therefore, there is a real need for all health professionals (i.e. health informatics professionals, physicians, nurses etc.) to learn about differing types of EHRs with differing content, interface designs, features and functions. Such opportunities to work with differing records are necessary so that health professionals can easily learn, adopt and adapt to differing types of EHRs.

Such work in a classroom and laboratory environment would lead to the development of health professionals that are not only technologically savvy, but understand the basic theoretical underpinnings of all EHRs and how they may be used to extract patient information that is relevant to their decision making. Such knowledge would ease adoption and facilitate uptake of EHRs as well as new content, interface designs, features and functions of EHRs over time (Borycki et al., 2009). Before this can happen, there is a need to develop health professional faculty EHR competencies, address health informatics professional shortages, and provide health professional students with platforms for providing students with opportunities to work with representative examples of these types of systems any time and any place.

\section{Overcoming Educational Barriers: Design and Development of a Web- based Tool}

As a way of overcoming the barriers described above, several researchers at the University of Victoria have been involved in the development of a Web-based EHR portal. This research, which led to the development of a unique portal, allows health professional students and faculty to access and learn about a range of EHRs over the WWW. Differing EHRs are available on the portal and provide health professional students and faculty with opportunities to learn about them. These EHRs have differing design metaphors, features and functions. These EHRs have given students and faculty the opportunity to learn about the impacts of EHRs upon clinical practice and have allowed faculty to integrate these records into health professional student education. 
Students have worked with differing types of EHRs through the portal prior to working in real-world settings. The portal provides faculty and students with a high-fidelity simulated EHR environment that is similar to the real-world. Patient data within the EHR is fictitious, but is realistic in nature. This allows students to better understand how patient data would be storied in differing EHRs.



Figure 1. Remote desktop of the UVic-EHR Portal, as seen by a student logging into the OpenVista ${ }^{\circledR}$ system remotely

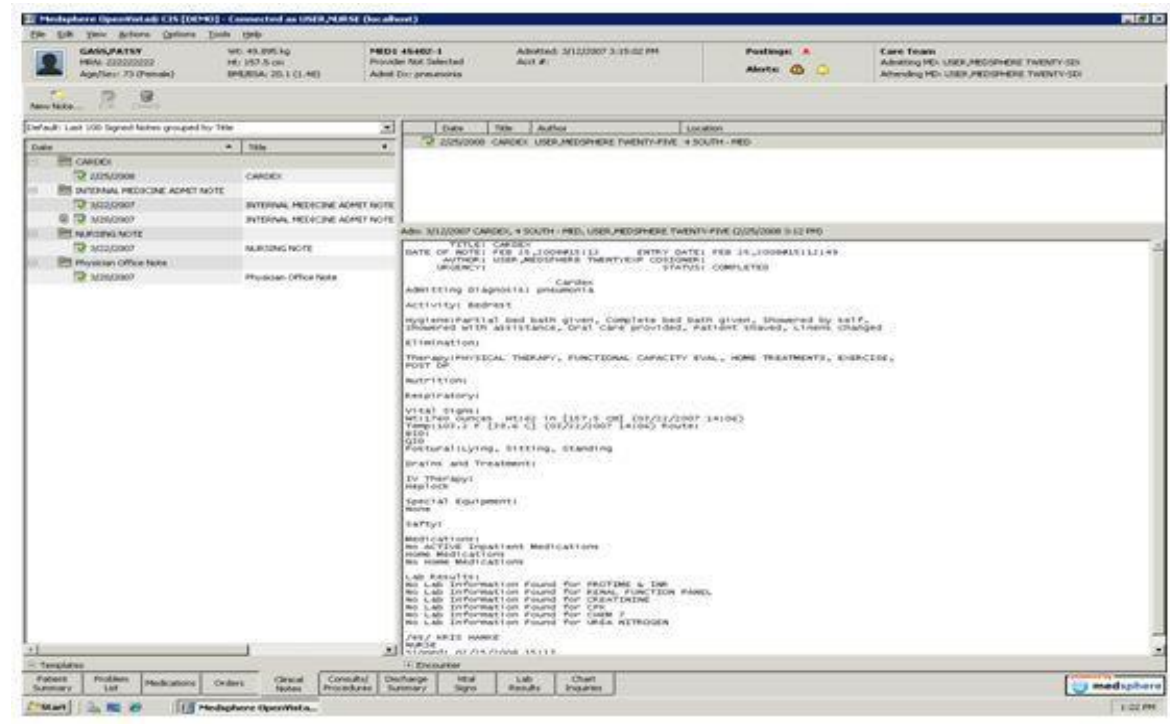

Figure 2. Student view of a fictitious patient record displayed in OpenVista ${ }^{\circledR}$

To illustrate student access to the portal, Figure 1 shows the first screen that a student would see if they accessed the EHR portal via the WWW. In Figure 1 the student has clicked on an icon and has started up an instance of the OpenVista ${ }^{\circledR}$ EHR. The remote desktop that a student $\log$ s onto is located on servers in Victoria, British Columbia, Canada. The use of a remote desktop provides every student private read and 
write access to a range of EHRs including EMRs, PHRs and EHRs. In Figure 2, the student has entered the EHR Open Vista ${ }^{\circledR}$ and is examining a fictitious patient record.

\section{Conclusion}

We have overcome a number of barriers to providing health professionals with educational opportunities to learn about EHRs. With the development of a web-based portal that provides students from the health disciplines with access to a range of EHRs over the WWW. We have also been able to provide health professional faculty with opportunities to work with differing types of EHRs while at the same time limiting our need to depend on a large number of health informatics specialists to provide access to EHRs (i.e. one portal can provide health professional students with access to many EHRs across the country and internationally). We have also provided students with access to differing types of EHRs that students would encounter over the course of a career. As a result, health professional faculty have developed an understanding of EHRs and have begun to integrate the portal into health professional student classroom work (e.g. Borycki et al., 2009). Faculty have developed differing educational approaches to teaching students about EHRs. In the classroom and in the laboratory setting students have learned how to use this technology in a "safe" environment where there are no direct impacts on patient care or implications for health professional practice. Students have also learned about the strengths and limitations of the technology. For example, exposure to differing EHRs has stimulated discussion with students about the ways in which the technology can reduce error rates as well as introduce new types of errors (e.g. technology-induced) errors.

We have begun to overcome the barrier of faculty competencies where EHRs are concerned by providing faculty with access to the portal (by making the web link to the portal open and accessible from anywhere and any place). We have begun to overcome the barrier of a shortage of health informatics specialists needed to implement such systems. This is achieved by having a centralized, Web-accessible portal that not only provides access to real working systems, but can be accessed locally and globally (simply by logging on to the portal web site). Victoria based students have been able to access portal EHRs in local university classrooms and distance-education students from other parts of Canada and internationally have be able to access portal EHRs over the WWW.

Prior to this, schools and universities interested in exposing students to this type of technology typically had to hire staff to either install and maintain a local system that could only be used in a local university or college computer lab, or via a link to a local hospital-based information system (note that the University of Victoria portal only contains fictitious patient data, making the EHRs and the data contained within them on the portal accessible worldwide without privacy concerns).

This approach (i.e. a web-based EHR portal approach) reduces the costs associated with EHR access for students and faculty (i.e. some local installations of commercial systems in a single educational institutions can have costs in the several thousands of dollars whereas the portal is accessible worldwide over the WWW for nominal cost to many universities). In addition to this, the barrier to exposing students to more than one system has been overcome by this work, since the portal currently contains multiple open source plus commercial EHR systems. Thus faculty and instructors can teach students about many types of EHRs by via the portal. A limitation of the portal is that it does not obviously contain all types of EHR systems. However, the EHRs that have been deployed on the portal have been judged by a panel of several 
health informatics experts to be highly representative of types of EHRs that health professional student graduates will be expected to understand and work with once they graduate and begin to practice in the real-world. Current work along these lines has involved close collaboration with a number of medical, nursing and health informatics programs worldwide to soundly integrate use of real technology into the basic education of these future professionals.

\section{Acknowledgements}

The work described in this paper was supported through a research grant from the British Columbia Ministry of Health (with hardware support from Hewlett-Packard).

\section{References}

1. Armstrong, B., Kushniruk, A.W., Joe, R., \& Borycki, E. (2009). Technical and architectural issues in deploying electronic health records (EHRs) over the WWW. Studies in Health Technology and Informatics, 143, 93-98.

2. Borycki, E.M., Kushniruk, A.W., Joe, R., Armstrong, B., Otto, T., Ho, K., Silverman, H., Moreau, J. \& Frisch, N. (2009). The University of Victoria Interdisciplinary Electronic Health Record Educational Portal. Studies in Health Technology and Informatics, 143, 49-54.

3. Borycki, E.M. \& Kushniruk, A.W. (2008). Where do technology-induced errors come from? Towards a model for conceptualizing and diagnosing errors caused by technology (pp. 148-166). In A. W. Kushniruk and E. M. Borycki (Eds.). Human Social and Organizational Aspects of Health Information Systems. Hershey, Pennsylvania: Idea Group.

4. Borycki, E.M. \& Kushniruk, A.W. (2010). Towards an integrative cognitive-sociotechnical approach in health informatics: Analyzing technology-induced error involving health information systems to improve patient safety. Open Medical Informatics Journal, 4, 181-187.

5. Canada's Health Informatics Association. (2009). Health informatics and health information management: Human resources report. Toronto: author.

6. Connors, H.R., Weaver, C., Warren, J. \& Miller, K.L. (2002). An academicbusiness partnership for advancing clinical informatics. Nursing Education Perspectives, 23(5), 228-233.

7. Dess, G.G., Lumpkin, G.T., Eisner, A.B. \& Perdis, T. (2009). Strategic management: Creating competative advantages. McGraw-Hill Ryerson.

8. Issenberg, S.B., McGaghie, W.C., Hart, I.R., Mayer, J.W., Felner, J.M., Petrusa, E. R., Waugh, R.A., Brown, D.D., Safford, R.R., Gessner, I.H., Gordon, D.L. \& Ewy, G.A. (1999). Simulation technology for health care professionals skills training and assessment. JAMA, 282(9), 861-866.

9. Koppel, R., Metlay, J.P., Cohen, A., Abaluck, B., Localio, A.R., Kimmel, S.E., \& Strom, B. L. (2005). Role of computerized physician order entry systems in facilitating medication errors. JAMA, 293(10), 1197-203.

10. Kushniruk, A.W., Borycki, E., Kuwata, S. \& Kannry, J. (2005). Predicting changes in workflow resulting from healthcare information systems: Ensuring the safety of healthcare. Healthcare Quarterly, 9S, 114-118. 
11. Kushniruk, A.W., Triola, M.M., Borycki, E.M., Stein, B. \& Kannry, J.L. (2005). Technology-induced error and usability: The relationship between usability problems and prescription errors when using a handheld application. International Journal of Medical Informatics, 74, 519-526.

12. Kushniruk, A.W. \& Borycki, E.M. (2008). Human, social and organizational aspects of health information systems. Hershey, Pennsylvania: IDEA Group.

13. Kuziemsky, C.E., Borycki, E.M., Purkis, M.E., Black, F., Boyle, M., CloutierFisher, D., Fox, L. A., MacKenzie, P., Syme, A., Tschanz, C. Wainwright, W. \& Wong, H. (2009). An interdisciplinary team communication framework and its application to healthcare e-teams systems design. BMC Medical Informatics and Decision Making. Sept 15, 9-43.

14. Nagle, L. (2007). Informatics: Emerging concepts and issues. Nursing Leadership, 20(2), 30-33.

15. Nohr, C., \& Boye, N. (2008). Towards computer supported clinical activity: A roadmap based on empirical knowledge and some theoretical reflections (pp. 6783). In A. W. Kushniruk and E. M. Borycki (Eds.). Human Social and Organizational Aspects of Health Information Systems. Hershey, Pennsylvania: Idea Group.

16. Otto, A., \& Kushniruk, A.W. (2009). Incorporation of medical informatics and information technology as core components of undergraduate medical education Time to change! Studies in Health Technology and Informatics, 143, 62-67.

17. Patel, V.L., Kushniruk, A.W., Yang, S. \& Yale, J-F. (2001). Impact of a computerbased patient record system on data collection, knowledge organizations and reasoning. JAMIA, 7, 569-585.

18. Popkin, J., Kushniruk, A.W., Borycki, E., Guarin, D., Mozley, L., Kilarsky, N., Robson, L. \& Creed, W. (2009). The eFOSTr Project: Design, implementation and evaluation of a web-based personal health record to support health professionals and families of children undergoing transplants. Studies in Health Technology and Informatics, 143, 358-363.

19. Protti, D. (2007). Comparison of information technology in general practice in ten countries, Healthcare Quarterly, 10(2), 107-116.

20. Shortliffe, E.H. \& Cimino, J.J. (2008). Biomedical informatics: Computer applications in health care and biomedicine. New York: Springer Verlag.

21. World Health Organization. (2005). eHealth tools and services: Needs of the member states. World Health Organization: Geneva. 